\title{
Reseña. Handbook of Research on Citizenship and Heritage Education, por Emilio José Delgado Algarra y José María Cuenca López (Coords.)
}

IGI Global, 623 pp. $212 €$ (tapa dura), 132,50€ (ebook), 9781799819783, https://www.igi-global.com/book/handbookresearch-citizenship-heritage-education/234655

Daniel Abril-López

Universidad de Alcalá

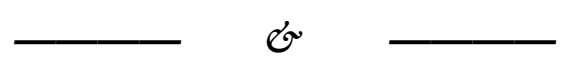

Este libro proporciona una visión general, desde una perspectiva internacional, de la educación patrimonial y para la ciudadanía. Defiende una visión interdisciplinar, holística, sistémica, constructivista, participativa, interactiva, compleja y sociocrítica (Cuenca et al., 2017).

Recoge trabajos de Alemania, Canadá, Chile, China, España, Estados Unidos, Hong Kong, Italia, Reino Unido y Singapur. Aborda fundamentos teóricos y curriculares, procesos de investigación actuales, y un conjunto de experiencias prácticas que analizan aspectos didácticos desde el campo educativo formal y no formal. Todo ello dentro de las diferentes temáticas incluidas por Fontal e IbáñezExteberria (2017): educación, comunicación, difusión, interpretación y enseñanza del patrimonio; museografía y musealización didáctica; patrimonialización; e identización. 
Los capítulos 1-9 están incluidos en la primera de las tres secciones, titulada Theoretical Reflections, Education Policies, and Curricula. El primer capítulo, Challenges for the Construction of Identities With Historical Consciousness (Delgado-Algarra y Cuenca-López), analiza bases de datos para conocer cómo se trabaja la educación ciudadana y patrimonial; describe experiencias didácticas; y promueve nuevas líneas de investigación vinculadas con la transversalidad, problemáticas socioambientales, justicia social, empatía sociocultural, y construcción de identidades.

Critical Citizenship Education and Heritage Education (cap. 2, SantistebanFernández, González-Monfort y Pagès-Blanch), considera que la educación patrimonial es una herramienta extraordinaria para ayudarnos a comprender el cambio y la continuidad de ideas, tradiciones y aspectos de la vida cotidiana; favorecer la conciencia crítica de la juventud para que reinterprete su cultura en base a su realidad e intereses; y crear interrelaciones pasado-presente-futuro.

Heritage in Conflict (cap. 3, Estepa-Giménez y Martín-Cáceres), tiene como objetivo dotar a los estudiantes de contenidos, habilidades, destrezas y competencias características del pensamiento crítico. La educación, según los autores, debe beneficiar metodologías activas, la participación social y la sostenibilidad de nuestro planeta.

Por su parte, Citizenship and Social Studies Curricula in British Columbia, Canada (cap. 4, Broom), se centra en el análisis de la última revisión curricular sobre la educación para la ciudadanía del estado canadiense de Columbia Británica. Para la autora, dentro de los estudios sociales, el objetivo es crear "good citizens" (p. 71) en defensa de la democracia y el multiculturalismo.

Heritage Education and Global Citizenship (cap. 5, González-Valencia, Massip y Castellví), persigue una educación desde una perspectiva sociocrítica más allá de la interrelación tradicional entre educación patrimonial e historia del arte. Los autores se decantan por el fomento de la identidad (global) y la participación ciudadana (crítica y comprometida).

Heritage and Museums as Objects of Education for Citizenship in the Teaching of History (cap. 6, Escribano-Miralles, Miralles y Serrano-Pastor), proporciona premisas teóricas para trabajar en el aula con patrimonio arqueológico. A este respecto, los autores afirman que las salidas didácticas (por ejemplo, a instituciones museísticas), en cualquier etapa educativa, son poderosas herramientas para contribuir al aprendizaje significativo de las ciencias sociales. 
Heritage Education as an Instrument for the Construction of a Democratic Citizenship in the Social Sciences Classrooms of Secondary Education (cap. 7, Coma, Fuentes y Sánchez), se articula a través de dos escenarios educativos: la escuela (educación formal) y la ciudad (educación no formal), tomados como entornos con gran potencial para la defensa del concepto interdisciplinar-igualdad, ciudadanía, derechos humanos- de democracia. A este respecto, las autoras apoyan metodologías activas como el "learning by proyects (PBL)" (p. 137).

Arabic Language Heritage Schools (cap. 8, Szczepek, Bengsch, Said, Scally y Davies), discute temas clave sobre la identidad personal (individualidad) y social (colectividad). En este trabajo se esbozan recomendaciones que ayuden a lograr una respuesta educativa más sofisticada a la diferencia cultural, desde cinco parámetros: optimismo, asociación, conocimiento, evaluación y pedagogía.

Y The Heritage and Education Research Network (cap. 9, Fontal y Martínez), sugiere -ante las deficiencias percibidas- generar una red interdisciplinaria de múltiples agentes sobre la investigación de la educación patrimonial en diferentes países europeos: desde el nivel conceptual, el diseño de políticas, hasta la práctica e implementación.

Los capítulos 10-17 forman parte de la segunda sección, denominada Researches. Heritage Education as a Tool for Creating Critical Citizens (cap. 10, Ferreras-Listán, Pineda-Alfonso y Hunt-Gómez), explora las concepciones que los futuros docentes de educación secundaria tienen con respecto a los conceptos de ciudadanía, democracia y patrimonio, conducentes al fortalecimiento de una formación ciudadana activa y crítica que enfrente los desafíos de la ciudadanía global.

Heritage Education in Central China (cap. 11, On Lee, Hao y Zhou), proporciona un estudio de caso de educación patrimonial en la región de la Llanura Central, una de las cunas más importantes de la civilización; se reflexiona sobre la dimensión “inter-cultural” (p. 232) en los centros educativos; y cómo el aprendizaje inclusivo e interdisciplinario es fundamental en esta era de globalización.

Trainee Teachers' Perceptions of History Teaching and the Critical Education of Citizenship (cap. 12, Gómez Carrasco, López y Castro), aporta un estudio realizado en las universidades de Murcia y Santiago de Compostela con respecto a las percepciones históricas de los profesores en formación. Los resultados aportados por los autores transmiten un modelo crítico basado en valores éticos y justicia social; aunque necesitado de mejores conexiones teórico-prácticas, ya que el modelo implícito es aún tradicional en sus concepciones teóricas y metodológicas. 
El decimotercer capítulo, Heritage Education and Citizen Education in Chile (Muñoz, Vásquez Lara, Collao Donoso, Vásquez Leyton, Torres, Muñoz, Montanares y Gutiérrez Cortés), presenta los resultados de una investigación realizada en diez museos chilenos para describir y analizar el vínculo entre el museo y la escuela. Para ello, plantean preguntas relevantes para el afianzamiento de enfoques didácticos: qué y cómo se puede aprender en el museo; cuáles son los enfoques y propuestas; o qué vínculos existen entre el patrimonio y la educación ciudadana.

En Historical Memory in Heritage Education Apps, Gillate, Luna, Castrillo e Ibáñez-Etxeberría exploran aplicaciones internacionales destinadas a preservar la memoria histórica, y para promover la competencia social y cívica. Pero apenas se percibe "history with memory" (p. 303) con interpretación, análisis y crítica, vital en cualquier proceso de enseñanza y aprendizaje de las ciencias sociales.

El decimoquinto capítulo, Researches, Projects, and Experiences in Didactics of History and Heritage From the DiPaSt Center of the University of Bologna, Italy (Borghi y Galletti), presenta la participación de escuelas y docentes, y autoridades locales, en la didáctica de la historia y el patrimonio. Los autores proponen el desarrollo de un plan de estudios interdisciplinar entre la educación formal (educación infantil y primaria) e informal; y la elaboración de proyectos de investigación para la formación del profesorado que interrelacione educación patrimonial y ciudadanía activa.

Religious and Ethnic Identification of Minoritized Youth in Hong Kong: Exploring Acculturation Outcomes (cap. 16, Cheung, Bhowmik, Kennedy, Yu Ngy Tak Hue), explora los casos de jóvenes inmigrantes de segunda generación en Hong Kong. La juventud india parece tener un nivel bajo de identificación religiosa y étnica; mientras que la paquistaní muestra una afiliación religiosa fuerte y un grado relevante de pertenencia a su cultura patrimonial. La aculturación se percibe como un proceso dependiente del contexto, los valores, los compromisos y las prácticas grupales.

El decimoséptimo capítulo, Future Citizens: Analysis of the Knowledge and Attitudes on Citizenship of Elementary Education Pupils of Murcia, Spain, From an Inquiry Based on the ICCS (Molina y Riquelme), analiza los deficientes conocimientos, actitudes y valores de los estudiantes de sexto curso de Primaria (11-12 años) en educación cívica y ciudadanía, aun cuando son necesarios para una participación social activa. 
Los capítulos 18-23 se insertan en la tercera y última sección: Experiences and Didactic Proposals. El decimoctavo capítulo, The Difficulty of Teaching Historical Landscape: Observations Starting From the Italian Situation (Brusa), tiene como tarea el establecimiento de una didáctica sobre la educación del paisaje basada en grandes contextos espacio-temporales, e incluso literarios, y superar microhistorias, y en Democratic Learning Environment Formats for the Empowerment of Youth, Gantenberg y Partetzke persiguen el aumento del compromiso, la protección y la participación democrática de los jóvenes desfavorecidos en cinco países europeos, bajo el proyecto "European Learning Environment Formats for Citizenship and Democracy (ELEF)".

El vigésimo capítulo, The Spanish Civil War in the Classrooms (FeliuTorruella y Hernández-Cardona), conciencia sobre el potencial pedagógico de la historia de la Guerra Civil española, y su patrimonio, para desarrollar un programa de educación ciudadana para la paz y crítica, vivir dentro de la complejidad de nuestro mundo y proponer actividades con materiales patrimoniales.

Educommunication Web 2.0 for Heritage (cap. 21, Rivero, Navarro y Aso), examina cómo los grandes museos son capaces de generar contenido multiforme y atractivo, pero que apenas fomentan el intercambio de experiencias entre usuarios. Para los autores, las comunidades cibernéticas, lideradas por los "millennials" (p. 465), no sólo deben disponer de información patrimonial, sino de enfatizar su valor, hacerlo accesible, y de desplazar la acción desde las redes sociales hacia el mundo físico.

El vigesimosegundo capítulo, Conflictive Memory and Heritage Education in the Initial Training of Primary Teachers (Domínguez-Almansa, Riveiro-Rodríguez, Monteagudo-Fernández y López Facal), muestra que las competencias sociales y cívicas mejoran cuando se fomentan las aptitudes sociales (emoción, racionalidad). Siguiendo a los autores, mediante la reformulación de los modelos didácticos, trabajo colectivo, y nuevos proyectos y experiencias en educación patrimonial.

Finalmente, en Educating Multicultural Citizens From a Confucian Heritage (cap. 23, Pinhui Wu, Tan y Ng), sugiere una noción confuciana de ciudadanía multicultural y armonía interreligiosa en el sureste asiático: abierto a otras culturas (shu o empatía), y promoviendo valores universales (ren o humanidad).

Percibimos, tras la lectura de este libro, la defensa de varias cuestiones clave de la Educación actual: reforzar los valores democráticos y el estudio del patrimonio; averiguar la identidad simbólica que facilite la comprensión del presente y el 
pasado; y formar a una ciudadanía comprometida con el contexto próximo y global, emancipada, transformadora y con memoria (Delgado-Algarra y Estepa-Giménez, 2017).

El libro reseñado incluye capítulos muy enfocados a la adquisición y fomento de determinadas competencias: por ejemplo, la social y cívica (números 14 y 22). En la legislación española (ORDEN/ECD/5/2015) se menciona que las siete competencias clave (lingüística; matemática, y ciencia y tecnología; digital; aprender a aprender; sociales y cívicas; sentido de iniciativa y espíritu emprendedor; conciencia y expresiones culturales) deben desarrollarse en los ámbitos de la educación formal, no formal e informal a lo largo de toda la vida. La finalidad es lograr que los individuos alcancen un pleno desarrollo personal, social y profesional.

La competencia lingüística está recogida, implícitamente, en todos los capítulos. Elaborados en inglés por especialistas de unos diez países, varios trabajos incorporan experiencias vinculadas con la educación plurilingüe, la diversidad cultural, y el enriquecimiento de la ciudadanía: capítulo 1 («empatía sociocultural»); capítulo 4 («multiculturalismo»); capítulo 8 («diferencia cultural»); capítulo 9 ( «patrimonio europeo»); capítulo 11 («dimensiones interculturales»); capítulo 16 («identificación religiosa y étnica»); y capítulo 23 («ciudadanía multicultural»). Vinculado a lo anterior, el desarrollo del espíritu crítico se introduce de forma evidente en los capítulos número 2 («conciencia crítica»); número 3 («pensamiento crítico»); número 5 («perspectiva sociocrítica»); número 10 («ciudadanía crítica»); número 12 («modelo crítico»), y número 20 («memoria histórica crítica»).

El compendio de estos capítulos se articula, en definitiva, como un instrumento muy útil para determinar la situación existente y los problemas en las conexiones entre educación patrimonial y educación ciudadana, difundiendo líneas de acción desde perspectivas vigentes e innovadoras.

\section{REFERENCIAS}

Cuenca-López, J. M., Estepa-Giménez, J. Y., \& Martín-Cáceres, M. J. (2017). Patrimonio, educación, identidad y ciudadanía. Profesorado y libros de texto en la enseñanza obligatoria. Revista de Educación, 375, 136-159. http://doi.org/10.4438/1988-592X-RE-2016-375$\underline{338}$

Delgado-Algarra, E., \& Estepa-Giménez, J. (2017). Educación ciudadana y dimensiones de la memoria en la enseñanza de las Ciencias Sociales: investigación sobre las concepciones del profesorado de educación secundaria de Huelva y provincia. Educación XXI, 20(2), 259278. http://doi.org/10.5944/educXX1.19041

Fontal, O., \& Ibáñez-Etxeberria, A. (2017). La investigación en Educación Patrimonial. Evolución y estado actual a través del análisis de indicadores de alto impacto. Revista de Educación, 375, 184-214. http://doi.org/10.4438/1988-592X-RE-2016-375-340 
DANIEL ABRIL-LÓPEZ

Profesor Ayudante Doctor (UAH). Licenciado en Historia, y Doctor en Prehistoria. Tres másteres oficiales vinculados con el Patrimonio y la Educación. Premios Fin de Carrera, y Extraordinario de Doctorado. Docencia en Historia; Educación Infantil, Primaria y Social; y Máster. Autor o coautor de más de 30 publicaciones (JCR, SJR, etc.).

daniel.abril@uah.es https://orcid.org/0000-0002-5283-4192

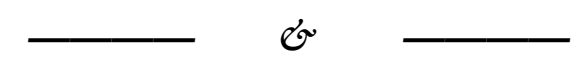

Abril-López, D. (2021). Reseña. Handbook of Research on Citizenship and Heritage Education, por Emilio José Delgado Algarra y José María Cuenca López (Coords.). Bellaterra Journal of Teaching \& Learning Language \& Literature, 14(2), e892. https://doi.org/10.5565/rev/jt13.892 